\title{
Induction chemotherapy before autologous stem cell transplantation for symptomatic plasma cell myeloma - does it matter?
}

This article was published in the following Dove Press journal:

Clinical Pharmacology:Advances and Applications

13 April 2010

Number of times this article has been viewed

\author{
Henry C Fung \\ Sunita Nathan \\ John J Maciejewski \\ Coleman Foundation Blood and \\ Marrow Transplantation Program, \\ Section of Bone Marrow Transplant \\ and Cell Therapy, Division of \\ Hematology/Oncology/Stem Cell \\ Transplant, Rush University Medical \\ Center, Chicago, Illinois, USA
}

\begin{abstract}
Autologous stem cell transplantation is the preferred treatment option for younger patients with symptomatic plasma cell myeloma. Most patients with newly diagnosed plasma cell myeloma receive 3-4 cycles of induction chemotherapy to achieve a level of disease control before proceeding to stem cell transplant. The ideal induction regimen for transplant-eligible patients shall allow more patients to proceed with transplant, rapidly and effectively control the disease, reverse disease-related complications, avoid early death, and is associated with minimal acute and long-term toxicities. Because of the concerns of potential damages to hematopoietic stem cells, alkylating agent regimens, specifically melphalan, are usually avoided for induction in transplant-eligible patients. Before the advance of immunomodulatory agents (IMiD) and proteasome inhibitors, the combination of vincristine, adriamycin, and dexamethasone (VAD) and variants were the most commonly used induction regimens. Recent reports as discussed in this review suggests that VAD is no longer the induction chemotherapy of choice for transplant eligible patients. Newer regimens incorporating IMiD and/or proteasome inhibitor into the induction regimen improve response rates and progression-free survival before and after the transplant and are evolving as the treatment of choice. Here, we review the available data on these newer induction regimens and to evaluate the potential impacts on the patient outcomes.
\end{abstract}

Keywords: plasma cell myeloma, induction chemotherapy, autologous stem cell transplantation

\section{Introduction}

Based on the results of two landmark studies published in the New England Journal of Medicine in 1996 and 2003, by the Intergroupe Francophone de Myeloma (IFM), France, ${ }^{1}$ and Medical Research Council (MRC), United Kingdom, ${ }^{2}$ respectively, upfront autologous stem cell transplant (ASCT) is considered the preferred treatment option for younger patients with newly diagnosed plasma cell myeloma. Practice guidelines from both sides of the Atlantic endorse this treatment approach, ${ }^{3,4}$ although the definition of "young," and hence transplant eligibility, differs. In Europe patients aged 60-65 years are usually considered to be eligible for transplant, but there is no upper age limit in the US. Although it is not the purpose of this review to address transplant eligibility, in our opinion, age alone should not be an exclusion criterion for ASCT. It is the responsibility of the treating physician to help patients establish their treatment goals and to discuss the risks and benefits of all treatment options available to them. It is also important for physicians and patients to be aware of the impact that certain induction regimens may have on potential stem cell harvest, which may affect eligibility for a future transplant.
Correspondence: Henry C Fung Rush University Medical Center, Section of Bone Marrow Transplant and Cell Therapy, 1725 West Harrison Street, Suite 834, Chicago, IL 606I2, USA 
Most patients with newly diagnosed plasma cell myeloma are treated with 3-4 cycles of induction chemotherapy in an attempt to achieve a level of disease control before proceeding to stem cell transplant. Induction chemotherapy is also intended to relieve symptoms, correct hypercalcemia, improve renal function, and improve performance status in preparation for a transplant. Before the introduction of novel agents, such as immunomodulatory agents and proteasome inhibitors, induction chemotherapy has not been shown to improve outcomes in patients undergoing ASCT. In fact, for patients with primary progressive plasma cell myeloma, multiple induction attempts are discouraged, since favorable outcomes with autologous stem cell transplant have been demonstrated in this setting. ${ }^{5}$ In the era before novel therapies, complete responses were uncommon, thereby making induction regimens less important and less relevant. The ideal induction regimen for a transplant-eligible patient should allow more patients to proceed with transplant. It should rapidly and effectively control disease, reverse disease-related complications, decrease the risk of early death, be easily tolerated with minimal/manageable acute and long term toxicities, and not interfere with the ability to harvest and collect stem cells for future stem cell transplantation. Furthermore, the regimen should improve response rates before and after ASCT and ultimately improve the progressionfree survival (PFS) and overall survival (OS).

\section{Melphalan-prednisone and vincristine-adriamycin- dexamethasone}

The combination of melphalan-prednisone (MP) had been the standard induction regimen for many decades until the advent of ASCT. ${ }^{6}$ However, the regimen has fallen out of favor because of the concern that exposure to alkylating agents can adversely affect stem cell harvest. Although a few cycles of MP probably will not preclude a successful harvest, newer induction regimens which appear to have faster and higher response rates when compared with MP should be considered (see below) in transplant-eligible patients.

Before the advances in novel agents such as immunomodulating drugs (IMiDs) and proteasome inhibitors (bortezomib), vincristine-doxorubicin-dexamethasone (VAD) was the most commonly used induction regimen for transplant-eligible patients. ${ }^{7}$ In VAD, the chemotherapeutic agents are given as a continuous intravenous infusion which, in addition to being inconvenient, also exposes patients to catheter-related complications. Rifkin et al substituted Doxil ${ }^{\circledR}$ for doxorubicin (DVD) and demonstrated a similar efficacy and toxicity profile to VAD. ${ }^{8}$ Since the activity of the VAD and DVD regimens is thought to be primarily because of the high-dose dexamethasone component, some hematologists and oncologists use dexamethasone alone as a "safer" and "better tolerated" alternative induction therapy for plasma cell myeloma. ${ }^{9}$ The typical high-dose dexamethasone using as a single agent (40 mg orally days $1-4,9-12,17-20$ ) is associated with lower response rates (approximately $45 \%$ ) when compared with VAD and DVD, without significant improvement in toxicity profile. In a randomized study comparing dexamethasone with thalidomide-dexamethasone (TD), the authors reported an early mortality of $10 \%$ which underscores the true toxicity of high-dose dexamethasone. ${ }^{10}$ The investigators from the European Cooperative Group for Bone and Marrow Transplantation (ECOG) reported similar results in another Phase III study comparing high-dose dexamethasone with lenalidomide/low-dose dexamethasone. ${ }^{11}$

\section{Thalidomide-dexamethasone}

In the early 2000s, a novel oral regimen using the combination of thalidomide-dexamethasone was introduced. The initial Phase II studies demonstrated response rates of $64 \%-76 \%$ which compared favorably with VAD. ${ }^{12}$ Because the combination yielded improved response rates and offered the advantage of an oral regimen, TD soon emerged as the preferred induction regimen for transplant-eligible patients. In a subsequent Phase III randomized study conducted by ECOG comparing $\mathrm{T}$ with $\mathrm{TD} ;{ }^{10}$ the best response rate with four cycles of therapy was $63 \%$ versus $41 \%$, respectively $(P=0.0017)$. In another confirmatory study that included 470 patients with newly diagnosed myeloma, TD was compared with placebodexamethazone (PD). ${ }^{13}$ According to the European Bone and Marrow Transplant (EBMT) response criteria, $63 \%$ of patients randomized to the TD arm achieved a complete response (CR) + a partial response (PR) compared with $46 \%$ in the PD arm $(P<0.001)$. Based on the International Myeloma Working Group criteria, $43 \%$ achieved CR/very good partial response (VGPR) in the TD arm compared with $15.8 \%$ in the PD arm $(P<0.001)$ with the median time to best response of 8.3 weeks versus 20.1 weeks, respectively. Notably, TD had no significant effect on stem cell yield. These two randomized studies further supported the practice of using thalidomide-dexamethasone as the induction regimen of choice in transplant-eligible patients, although it is not a "benign" regimen. In the ECOG study, 17\% patients in the TD arm developed deep vein thrombosis, compared with $3 \%$ in the control arm. Furthermore, Grade 3 or higher nonhematologic toxicities were seen more commonly in the TD arm $(67 \%$ vs $43 \%$ ). The early mortality (first four months) was $7 \%$ in 
the TD arm compared with $11 \%$ in the dexamethasone arm; although the TD arm was comparable with the dexamethasone arm, an early death rate of 7\% was still unacceptably high. Both studies, however, failed to demonstrate any improvement in time to progression, PFS, and OS after autologous stem cell transplantation, although these were not the primary endpoints. Macro et al reported a prospective study (albeit in abstract form), comparing TD with VAD, where $44 \%$ of patients in the TD arm achieved at least VGPR after ASCT, compared with $42 \%$ in the VAD arm. ${ }^{14}$ Of note, the use of TD was once again associated with a higher incidence of venous thromboembolism (VTE). Thus, taking into consideration the above data, we note that the improved response rates obtained with TD in comparison with VAD did not translate into improved outcomes after ASCT. Rather, the combination was associated with increased toxicities, thereby speaking against the routine use of thalidomide-dexamethasone as induction therapy prior to ASCT, given that the benefits when compared with VAD/DVD are, at best, marginal.

\section{Lenalidomide-dexamethasone}

Based on the assumption that lenalidomide is more potent and less toxic than thalidomide, investigators from the Mayo Clinic tested lenalidomide-dexamethasone in the upfront setting with $67 \%$ of patients achieving VGPR or better in a pilot study. ${ }^{15}$ Approximately, $50 \%$ of patients experienced Grade 3 or higher non-hematologic toxicity, similar to rates seen with dexamethasone alone. Clearly, this regimen needed improvement. In an attempt to reduce the toxicity of lenalidomide/ standard-dose dexamethasone, ECOG conducted a Phase III prospective study comparing lenalidomide/standard-dose dexamethasone with lenalidomide/low-dose dexamethasone (40 mg dexamethasone weekly). The toxicity rates were significantly higher with lenalidomide/standard-dose dexamethasone compared with lenalidomide/low-dose dexamethasone. ${ }^{11}$ Early mortality rates (first four months) were $5 \%$ versus $0.5 \%$, favoring the lenalidomidelow-dose dexamethasone arm. As with thalidomide, thromboprophylaxis is required for patients who received lenalidomide-containing regimens. Four hundred and forty-five patients were included in the study; 223 randomized to lenalidomide/standarddose dexamethasone and 222 to lenalidomide/low-dose dexamethasone. With a median followup of 17 months, OS was significantly superior with lenalidomide/low-dose dexamethasone $(P<0.001)$; one-year survival was $96 \%$ versus $87 \%$, respectively, and 18 -month survival $91 \%$ versus $80 \%$. OS differences in favor of the low-dose dexamethasone arm were seen in patients younger than 65 years $(P=0.022$; one-year survival rate $97 \%$ versus $92 \%$ ) as well as patients 65 years and older $(P=0.002$; one-year survival rate $94 \%$ versus $83 \%$ ), respectively.

Of some concern, recent reports suggest that prior exposure to lenalidomide may adversely affect stem cell mobilization kinetics. ${ }^{16}$ Thus, lenalidomide should be used cautiously in transplant-eligible patients, and stem cell harvest should be considered before the patients receive multiple cycles of lenalidomide. In a retrospective study of 472 patients, investigators from the Mayo Clinic did not find any differences in post-transplant outcomes in patients receiving VAD, dexamethasone alone, thalidomide-dexamethasone or lenalidomide-dexamethasone. ${ }^{17}$

In summary, the combination of lenalidomide and lowdose dexamethasone is an effective induction regimen which is well tolerated and associated with low early mortality. It should be considered as one of the options for transplanteligible patients with newly diagnosed symptomatic plasma cell myeloma. However, lenalidomide-dexamethasone has not been compared directly with VAD or a bortezomib-based induction regimen before ASCT. A Phase III prospective study will be required to define better the role of lenalidomide/low-dose dexamethasone as induction for transplanteligible patients.

\section{Thalidomide-doxorubicin- dexamethasone}

It is somewhat unfair to compare a two-drug regimen that contains thalidomide and dexamethasone with a three-drug regimen such as VAD, because doxorubicin is known to be an effective agent in the treatment of plasma cell myeloma. In a Phase III prospective study conducted by the Dutch Group (HOVON 50), thalidomide-doxorubicin-dexamethasone (TAD) was compared with VAD as induction therapy for transplant-eligible patients. ${ }^{18,19}$ Between November 2001 and May 2005, 556 patients were enrolled in the study. The primary endpoint was event-free survival (EFS). TAD yielded improved response rates when compared with VAD after ASCT (PR: $87 \%$ versus 79\%, $P<0.01$; VGPR: $65 \%$ versus $54 \%, P<0.01$; CR: $30 \%$ versus $21 \%, P=0.03)$. TAD also showed improved EFS (33 months versus 22 months; $P<0.001)$ and PFS (33 months versus 25 months; $P<0.001)$ when compared with VAD after ASCT. Because of a relatively short followup, OS benefit has not been demonstrated. In addition, with the availability of novel agents for salvage at the time of disease progression, it will be increasing difficult to show a survival benefit. Although the new combination of TAD compared favorably with VAD, regimens containing 
high-dose dexamethasone and/or thalidomide are associated with an increased risk of VTE, as well as the significant side effects from high-dose dexamethasone. One may argue that by using a lower dose of dexamethasone, similar to the ECOG study described earlier using lenalidomide/low-dose dexamethasone, ${ }^{11}$ the toxicity profile may improve. However, there are no good data to support this approach.

\section{Bortezomib-containing regimens}

In a Phase III randomized four-arm study (IFM 2005-01), Harousseau et al compared bortezomib-dexamethasone ( \pm dexamethasone-cyclophosphamide-etoposide-cisplatin [DCEP] consolidation) with VAD ( \pm DCEP consolidation). ${ }^{20}$ Patients received four cycles of VAD or bortezomibdexamethasone \pm two cycles of DCEP followed by a single cycle of high-dose melphalan $\left(200 \mathrm{mg} / \mathrm{m}^{2}\right)$ with autologous stem cell rescue. For patients who failed to achieve VGPR after the first transplant, a second autologous stem cell transplant, or reduced intensity allogeneic stem cell transplant, was considered. The response rates $(\mathrm{CR}+$ near $\mathrm{CR}, \geq \mathrm{VGPR}$ and $\geq$ PR) before and after ASCT all favored the bortezomibdexamethasone arm. The best responses after second autologous stem cell transplant were CR/near CR in $32 \%$ and $39 \%(P<0.001)$, VGPR in $47 \%$ and $68 \%(P<0.001)$ for the VAD arm versus the bortezomib-dexamethasone arm, respectively. The estimated two-year PFS was $60 \%$ in the VAD arm versus $69 \%$ in the bortezomib-dexamethasone $\operatorname{arm}(P=0.0115)$. Sonneveld et al compared three cycles of VAD with three cycles of bortezomib-adriamycindexamethasone (PAD) followed by ASCT. ${ }^{21}$ PAD delivered improved response rates compared with VAD. After the transplant, while $80 \%$ of patients who received PAD achieved VGPR; only $50 \%$ of patients who received VAD $(P=0.019)$ achieved a VGPR. Sixteen percent of patients developed Grade 3-4 neuropathy in the PAD arm versus 6\% in VAD arm. In a Phase III prospective, randomized study conducted by the Italian Multiple Myeloma Network ( GIMEMA), Cavo et al compared TD-ASCT with VTD-ASCT, which showed that response rates and PFS were superior in the VTD arm. ${ }^{22}$ On an intent-to-treat analysis, post-transplant $\mathrm{CR}$ rates were $41 \%$ in the VTD arm versus $20 \%$ in the TD $\operatorname{arm}(P<0.001)$. Two-year PFS was significantly superior with VTD compared with thalidomide-dexamethasone ( $90 \%$ versus $80 \%$, $P=0.009)$ but there was no difference in OS.

The above-mentioned studies, although reported in abstract form, suggest that bortezomib-based regimens compare favorably with VAD and TD. These novel regimens not only improve response rates before and after transplant, but have also demonstrated improvement in PFS, which is a strong surrogate marker for OS. In addition, multiple studies ${ }^{23-25}$ also suggest that bortezomib may be able to overcome the adverse prognostic effect of unfavorable cytogenetics. Furthermore, no dose adjustments are required for patients with renal insufficiency, even when they are receiving hemodialysis. In contrast, dose adjustment for lenalidomide is required for patient with renal insufficiency. It is also important to note that bortezomib-based regimens do not affect stem cell yields and thromboprophylaxis is not necessary. On the other hand, $10 \%-20 \%$ of patients treated with bortezomib will develop significant peripheral neuropathy. Nonetheless, data from large, well-controlled studies suggested that most patients with bortezomib-induced peripheral neuropathy improve or completely resolve at a median interval of three months.

\section{Future directions}

Recent reported Phase II studies that include both lenalidomide and bortezomib in multiagent regimens or sequential regimens have demonstrated $90 \%-100 \%>\mathrm{PR}, 60 \%-71 \%$ $>$ VGPR and $32 \%-36 \% \mathrm{CR} / \mathrm{nCR}^{26-28}$ In general, three or four cycles of induction chemotherapy are given before stem cell transplant. It is unclear whether a higher quality of response before stem cell transplant may be beneficial or may lead to increased transplant-related toxicity. In patients with acute leukemia, stem cell transplant is usually performed after achievement of remission, in whom the outcomes are more favorable than in patients who are transplanted with active disease. Although utilizing stem cell transplant as consolidation, similar to that employed in patients with acute leukemia and lymphoma, makes absolute sense, the role of stem cell transplant in patients with plasma cell myeloma who have achieved a complete remission is currently unknown. These important questions can only be answered in the context of a Phase III randomized trial.

\section{Conclusion}

In summary, outside of the setting of a clinical trial, transplant-eligible patients should be treated with 3-4 cycles of an induction regimen containing a novel agent before stem cell transplant. Examples include lenalidomide/low-dose dexamethasone, bortezomib-dexamethasone, PAD or VTD. For patients with high risk disease, particularly associated with unfavorable cytogenetics, the current literature favors the use of a bortezomib-based regimen, with or without an IMiD. For patients with pre-existing neuropathy, bortezomib should be used with caution. For patients with a previous history of thrombosis, or those at high risk of developing VTE, IMiD-containing regimens, although not absolutely 
contraindicated, should be avoided, especially given that other effective options are available.

\section{Acknowledgments}

Part of this work is funded by Coleman Foundation, Chicago, IL, USA. HF served on the following Speaker's Bureaus: Millennium, The Takeda Oncology Company, and OrthoBiotech Products, LP. The authors report no conflicts of interest in this work.

\section{References}

1. Attal M, Harousseau JL, Stoppa AM, et al. A prospective, randomized trial of autologous bone marrow transplantation and chemotherapy in multiple myeloma. N Engl J Med. 1996;335(2):91-97.

2. Child JA, Morgan GJ, Davies FE, et al. High dose chemotherapy with hematopoietic stem cell rescue for multiple myeloma. $N$ Engl J Med. 2003;348(19):1875-1883.

3. Smith A, Wisloff F, Samson D. Guidelines on the diagnosis and management of multiple myeloma. Br J Haematol. 2006;132(4):410-451.

4. Anderson KC, Alsina M, Bensinger W, et al. Multiple myeloma: Clinical practice guidelines in oncology. J Natl Compr Canc Netw. 2007;5(2):118-147.

5. Kumar S, Lacy MQ, Dispenzieri A, et al. High-dose therapy and autologous stem cell transplantation for multiple myeloma poorly responsive to initial therapy. Bone Marrow Transplant. 2004;34(2):161-167.

6. Myeloma Trialists' Collaborative Group. Combination chemotherapy versus melphalan plus prednisone as treatment for multiple myeloma: An overview of 6,633 patients from 27 randomized trials. J Clin Oncol. 1998;6(12):3832-3842.

7. Alexanian R, Barlogie B, Tucker S. VAD-based regimens as primary treatment for multiple myeloma. Am J Hematol. 1990;33(2):86-89.

8. Rifkin RM, Gregory SA, Mohrbacher A, Hussein MA. Pegylated liposomal doxorubicin, vincristine, and dexamethasone provide significant reduction in toxicity compared with doxorubicin, vincristine, and dexamethasone in patients with newly diagnosed multiple myeloma: A Phase II multi-center randomized trial. Cancer. 2006;106(4):848-858.

9. Alexanian R, Dimopoulos MA, Delasalle K, Bargolie B. Primary dexamethasone treatment of multiple myeloma. Blood. 1992;8(4):887-890.

10. Rajkumar SV, Blood E, Vesole D, et al. Phase III clinical trial of thalidomide plus dexamethasone compared with dexamethasone alone in newly diagnosed multiple myeloma: A clinical trial coordinated by the Eastern Cooperative Oncology Group. J Clin Oncol. 2006;24(3):431-436.

11. Rajkumar SV, Susanna Jacobus, Natalie Callander, et al. A randomized trial of lenalidomide plus high-dose dexamethasone (RD) Versus lenalidomide plus low-dose dexamethasone ( $\mathrm{Rd}$ ) in newly diagnosed multiple myeloma (E4A03): A trial coordinated by the Eastern Cooperative Oncology Group [Abstract]. Blood. 2007;110:74.

12. Rajkumar SV, Hayman S, Gertz MA, et al. Combination therapy with thalidomide plus dexamethasone for newly diagnosed myeloma. J Clin Oncol. 2002;20(21):4319-4323.

13. Rajkumar SV, Rosinol L, Hussein M, et al. Multi-center, randomized double blind placebo controlled study of thalidomide plus dexamethasone compared with dexamethasone as initial therapy for newly diagnosed multiple myeloma. J Clin Oncol. 2008;26(13):2171-2177.
14. Macro M, Divine M, Uzunhan Y, et al. Dexamethasone + thalidomide compared with VAD as a pre-transplant treatment in newly diagnosed multiple myeloma: A randomized trial [Abstract]. Blood. 2006;108:22a.

15. Lacy M, Gertz M, Dispenzieri A, et al. Lenalidomide plus dexamethasone in newly diagnosed myeloma: Response to therapy, time to progression, and survival [Abstract]. Blood. 2006;108:798

16. Kumar S, Dispenzieri A, Lacy MQ, et al. Impact of lenalidomide therapy on stem cell mobilization and engraftment post-peripheral blood stem cell transplantation in patients newly diagnosed multiple myeloma. Leukemia. 2007;21(9):2035-2042.

17. Kumar SK, Dingli D, Dispenzieri A, et al. Impact of pre-transplant therapy in patients with newly diagnosed myeloma undergoing autologous SCT. Bone Marrow Transplant. 2008;41(12):1013-1019.

18. Lokhorst H, Schmidt-Wolf I, Sonneveld P, et al. Thalidomide in induction treatment increase the very good partial response rate before and after high dose therapy in previously untreated multiple myeloma. Hematologica. 2008;93(1):124-127.

19. Lokhorst H, Van der Holt B, Zweegman, et al. Final analysis of HOVON-50 randomized Phase III study on the effect of thalidomide combined with adriamycine, dexamethasone and high dose melphalan in patients with multiple myeloma [Abstract]. Blood. 2008;112:157.

20. Harousseau JL, Mathiot C, Attal M, et al. Bortezomib/dexamethasone versus VAD as induction prior to autologous stem cell transplantation in previously untreated multiple myeloma; updated data from IFM 2005/01 trial. J Clin Oncol. 2008;26(15S):8505.

21. Sonneveld P, Van der Holt B, Schmidt-Wolf I, et al. First analysis of HOVON-65/GMMG-HD4 randomized Phase III trial comparing bortezomib, adriamycine, dexamethasone (PAD) versus VAD as induction treatment prior to high dose melphalan in patients with newly diagnosed multiple myeloma [Abstract]. Blood. 2008;112:653a.

22. Cavo, M, Tacchetti P Patriarca F, et al. Superior complete response rate and progresssion free survival after autologous stem cell transplantation with up-front velcade-thalidomide-dexamethasone compared with thalidomide-dexamethasone in newly diagnosed multiple myeloma [Abstract]. Blood. 2008;112:158.

23. Mateos MV, Hernandez JM, Hernandez MT, et al. Bortezomib plus melphalan and prednisone in elderly untreated patients with multiple myeloma: Results of a multicenter Phase I/II study. Blood. 2006;108(7):2165-2172.

24. Jagannath S, Richardson PG, Sonneveld P, et al. Bortezomib appears to overcome the poor prognosis conferred by chromosome 13 deletion in Phase 3 and 3 trials. Leukemia. 2007;21(1):151-157.

25. Sagaster V, Ludwig H, Kaufmann, et al. Bortezomib in relapsed multiple myeloma: Response rates and duration of response are independent of a chromosome 13q-deletion. Leukemia. 2007;21(1):164-168.

26. Richardson PG, Lonial S, Jakubowiak A, et al. Lenalidomide, bortezomib and dexamethasone in patients with newly diagnosed multiple myeloma: Encouraging efficacy in high risk groups with updated results of a phase I/II study [Abstract]. Blood. 2008;112:92a.

27. Kumar S, Flinn IW, Noga SJ, et al. Safety and efficacy of novel combination therapy with bortezomib, dexamethasone, cyclophosphamide, and lenalidomide in newly diagnosed multiple myeloma; initial results from phase I/II multi-center EVOLUTION study [Abstract]. Blood. 2008;112:93a.

28. Bensinger W, Jagannath S, Vescio R, et al. A Phase II study of bortezomib (Velcade $\left.{ }^{\circledR}\right)$, cyclophosphamide $\left(\right.$ Cytoxan $\left.^{\circledR}\right)$, thalidomide (Thalomid $^{\circledR}$ ) and dexamethasone as first-line therapy for multiple myeloma [Abstract]. Blood. 2008;112:94a.

\section{Dovepress}

\section{Publish your work in this journal}

Clinical Pharmacology: Advances and Applications is an international, peer-reviewed, open access journal publishing original research, reports, reviews and commentaries on all areas of drug experience in humans. The manuscript management system is completely online and includes a very quick and fair peer-review system, which is all easy to use.

Visit http://www.dovepress.com/testimonials.php to read real quotes from published authors. 